\title{
Clinical, Tricoscopic and Histopathological Findings in Mexican Women with Traction Alopecia
}

Martínez Suarez $\mathrm{H}^{1 *}$, Barrera Jacome $\mathrm{A}^{2}$, Ramirez Anaya $\mathbf{M}^{3}$, Barron Hernandez $\mathrm{L}^{4}$ and Morales Miranda $\mathrm{AY}^{5}$

${ }^{1}$ Dermatologist, Private practice in Marsu Dermatologia, Mexico

${ }^{2}$ Dermatopathologist, Deparment of Dermatology, Medical Specialties Unit, University of the Army and Air Force, Mexico

${ }^{3}$ Pediatric dermatologist, General Hospital in Cholula, Mexico

${ }^{4}$ Dermatologist and Dermatopathologist, Private practice in Puebla, Mexico

${ }^{5}$ Dermatologist, Deparment of Dermatology, Medical Medical Specialties Unit, University of the Army and Air Force, Mexico

*Corresponding author: Martínez-Suarez Hugo, Dermatologist, Private practice in Marsu Dermatologia, 7 Sur 3118 Chula Vista, Puebla, Zip: 72420, Mexico, Tel: 522225052798; Email: dermahugo@yahoo.com.mx

\section{Abstract}

Traction alopecia is a common form of hair loss in our population. It is caused by vigorous straightening of the hair causing areas of alopecia. We studied 43 patients diagnosed with traction alopecia from a clinical, tricoscopic and histopathological guide.

This disease has a great diversity of clinical findings and can also vary depending on the time of evolution. Histopathology is of great tool in case of diagnostic doubt and provides relevant data related to the evolution time. We believe that the population should change some hair styles to avoid progression to scarring alopecia.

Keywords: Traction alopecia; Cicatricial alopecia; Marginal alopecia; Trichoscopy; Fringe sign

\section{Introduction}

Traction alopecia is the hair loss caused by continuous, vigorous and chronic tightening of the hair. It is a very common disease in African-American women [1]. However, in Latino and Asian populations, it is more frequent than previously thought.

In each country and region, hair styling and hair care styles are dictated and influenced by sociocultural, occupational, and religious issues. So, in the black community, braids, weaves and dreadlocks are used with more frequency, in addition to the use of chemical smoothing and relaxers sustances. In Mexico and Latin America the use of ponytails is widespread. In India and Turkey the use of turbans predominates. All are worn for long periods of time a day for months or years [1-3].

This condition is not new, since there are descriptions that identify it for more than 100 years. Trebitsch, a dermatologist of Austrian origin, is apparently the first to describe it. Similarly, in the last century, some writings alluding to this disease were published, with data that currently continues to be valid.

The pathophysiology, it has not been fully identified. 


\section{Clinical Dermatology Open Access Journal}

The main cause known is the constant and strong pulling or traction of the hair. The hair is damaged when the hair fiber is tightened or stretched throughout its extension, for prolonged periods [2].

These different hairstyle styles give rise to diverse clinical forms. That depend on the evolution time and can be different in initial and late stages, even in the same patient [3].

The most commun clinical characteristics are: decreased capillary density in the marginal zone of the hair, alopecic plaques with the presence of hairs, peri and interfollicular erythema, as well as the described "fringe sign" [4].

Theoretically, traction alopecia is not difficult to identify. However, the diagnosis can be a true challenge. Especially, if the patient does not provide the information about the hairstyle she uses or event deny to use it. It is often confused with other hair disorders such as alopecia areata or frontal fibrosing alopecia due both can share some clinical characteristics. It is important to mention that this kind of alopecia does not provide much symptomatology, especially in its initial stages.

Trichoscopy is an important tool for the diagnosis of hair diseases. And in this condition it becomes essential, because allowed to identify if is an initial or advanced stage. And allows to identify if it is a scarring or non-scarring alopecia [5].

Although it is a frequent cause of alopecia, there are few studies about their clinical and trichoscopic characteristics. Most of the publications are isolated case reports.

\section{Material and Methods}

Retrospective, descriptive and cross-sectional review. We reviewed the clinical records of patients with traction alopecia diagnosed for two dermatologist in Mexico City and Puebla, México. Along one year (January 2019 to January 2020). The diagnoses was established with the clinical and trichoscopic findings. If the diagnosis was doubtful, we perfomed a biopsy per $4 \mathrm{~mm}$ cylinder, carrying out two cuts: one longitudinal and transverse, in both cases staining with hematoxylin and eosin. The variables analyzed were: age, occupation, educational level, time of use of hairstyle (years), also clinical characteristics as erythema, follicular papules, follicular pustules, peripillary cylinders, vellous hairs and alopecic patches). Trichoscopic findings: peripheral erythema, peripheral brown pigmentation, peripillary casts, black dots, broken hairs of different lengths and empty follicular openings. Clinical photographs were taken in 3 projections (frontal and bilateral) and trichoscopic of the involved areas. We used descriptive statistics.

\section{Results}

Forty-three women, aged 16 to 56 years (mean $33.9 \pm$ ) were included. Educational level was: (30\%) high school, (30\%) bachelor, $(27 \%)$ middle high school, (9\%) primary, (2\%) studies at master's level. Regarding the occupation we found: (45\%) housewives, $(16 \%)$ nurses, $(15 \%)$ teachers or educators, (7\%) others (employees, merchants, secretaries), (13\%) students (Table 1).

The range in the evolution time was from 1 to 35 years, with a mean of 16 years. $16(37 \%)$ of them manifested less than 10 years of evolution and $27(63 \%)$ more than 10 years with the problem.

Regarding the clinical characteristics, we observed: decrease in density or alopecic plaques in $100 \%$, vellous hair in forty-two cases (97\%), thirty-five (79\%) with peripheral erythema, six cases with peripheral papules and pustules in two $(5 \%)$ patients (Table 2) (Figure 1).

The tricoscopic findings were: peripyllari cast in thirtyfive (79\%), black dots in twenty-eight (53\%), broken hairs at a different level in twenty-two (51\%), perifollicular brown pigment in thirty-three (77\%), empty follicular openings in twenty-six (60\%). As extra findings, we observed irregular white dots in eight (19\%), pilli torti in three $(7 \%)$ and trichorhexis nodosa in two patiens (5\%) (Table 3) (Figure 2).

As mentioned, we carried out a histopathological study in some cases with the intention of observing the changes reported in the literature which are dependent on chronicity and thus establish the differences. In the patients with less than ten years we observed: preserved sebaceous glands in six (75\%), a decrease in terminal hairs in all of them (100\%), fibrotic fibrous tracts in three (37\%), and sparse lymphocytic inflammation in seven (87\%) (Table 4) (Figure 3).

In chronic forms we observed decrease in follicular density in the $88 \%$, as well as an increase in the number of follicles in catagen / telogen. Two patients (25\%) presented a decrease in the terminal / hairy ratio. Trichomalacia, pigment casts, or peripheral / intrafollicular hemorrhage were not observed in any of the biopsies studied. Alteration of follicular architecture was observed in two patients (25\%). $100 \%$ of the patients presented preserved sebaceous glands, a peripheral inflammatory infiltrate and fibrous tracts (Figure 4). Two patients (25\%) presented peripheral fibrosis. Only one patient presented "naked" hair follicles (12.5\%) (Figure 5). 


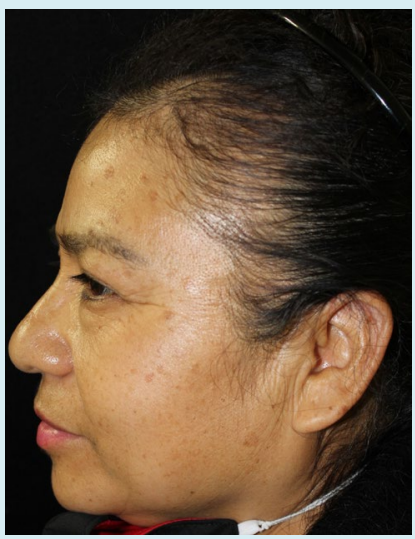

Figure 1: Women of 44 years old with traction alopecia.

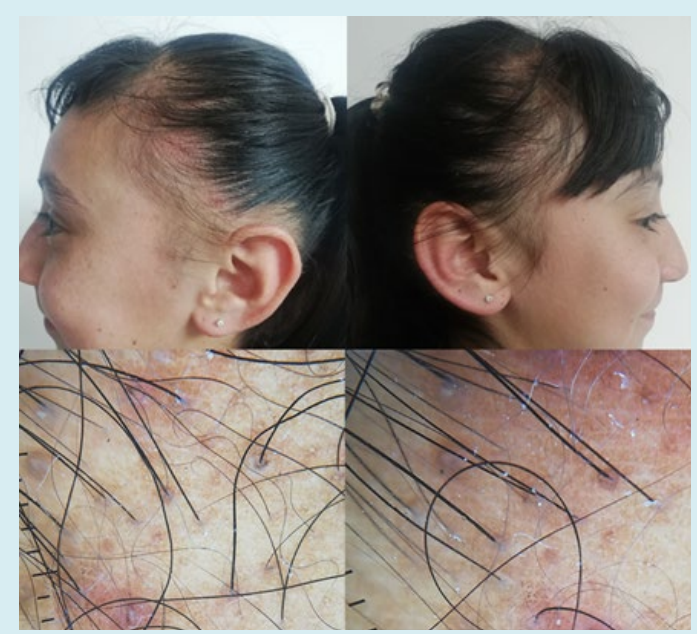

Figure 2: Girl of 16 years old, with alopecic patches located in bitemporal regions, we can see erythema and perifolcular papules. In the tricoscopy we can observe vellous hair, Black dots, peripheral marron pigmentation and hemorragic crust. Findings suggestive of traction folliculitis.

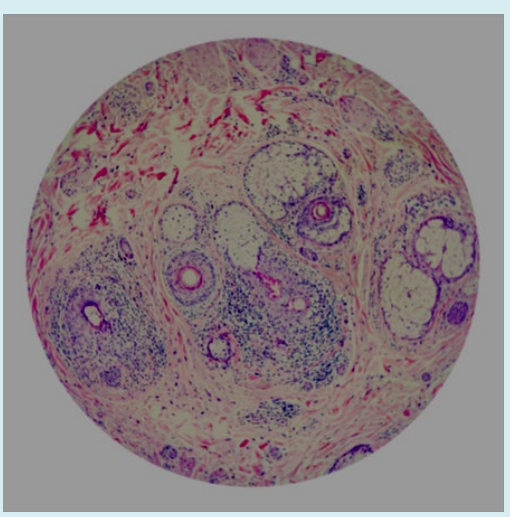

Figure 3: Preserved sebaceous glands and peripheral inflammatory infiltrate.
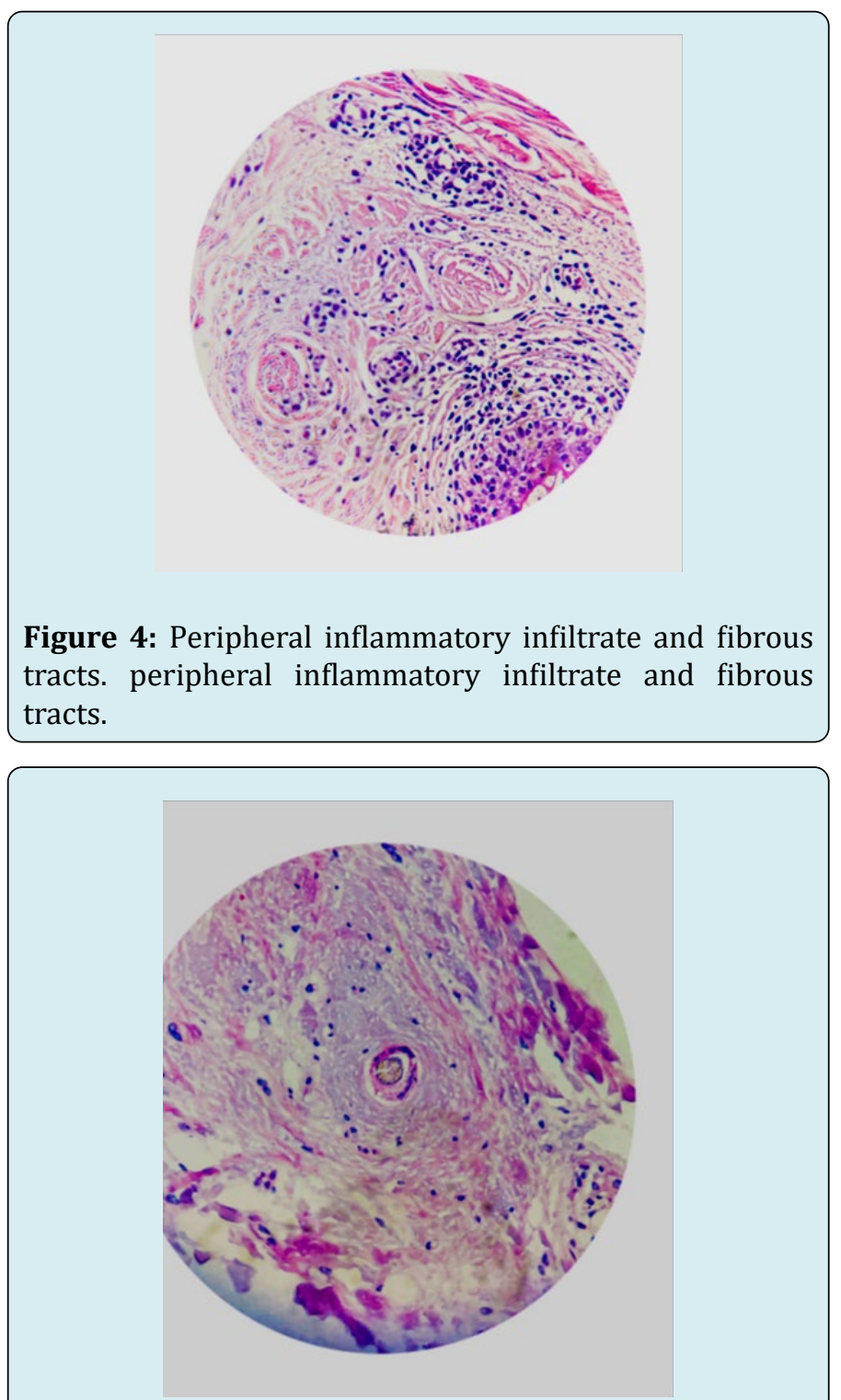

Figure 5: Naked hair follicles.

\begin{tabular}{|c|c|c|c|c|}
\hline $\begin{array}{c}\text { Level of } \\
\text { studies }\end{array}$ & Number & Percentage & Occupation & Percentage \\
\hline Bachelor & 13 & $30 \%$ & Housewives & $45 \%$ \\
\hline High school & 13 & $30 \%$ & Nurses & $16 \%$ \\
\hline $\begin{array}{c}\text { Middle high } \\
\text { school }\end{array}$ & 11 & $27 \%$ & Teachers & $15 \%$ \\
\hline Primary & 4 & $9 \%$ & Students & $13 \%$ \\
\hline Master level & 1 & $2 \%$ & Other & $7 \%$ \\
\hline
\end{tabular}

Table 1: 43 women, aged 16 to 56 years (mean $33.9 \pm$ ) and occupation. 


\begin{tabular}{|c|c|c|}
\hline Clinical Characteristics & $\begin{array}{c}\text { Number of } \\
\text { Patientes }\end{array}$ & $\begin{array}{c}\text { Percentage } \\
\text { (\%) }\end{array}$ \\
\hline Decrease in density & 43 & $100 \%$ \\
\hline Vellous hairs & 42 & $97 \%$ \\
\hline Peripheral erythema & 35 & $79 \%$ \\
\hline Peripheral papules & 6 & $13 \%$ \\
\hline Pustules & 2 & $5 \%$ \\
\hline
\end{tabular}

Table 2: Clinical characteristics.

\begin{tabular}{|c|c|c|}
\hline Tricoscopic findings & $\begin{array}{c}\text { Number of } \\
\text { patients }\end{array}$ & $\begin{array}{c}\text { Percentage } \\
\text { (\%) }\end{array}$ \\
\hline Peripyllari cast & 35 & $79 \%$ \\
\hline Black dots & 28 & $53 \%$ \\
\hline Broken hairs & 32 & $51 \%$ \\
\hline $\begin{array}{c}\text { Perifollicular brown } \\
\text { pigment }\end{array}$ & 33 & $77 \%$ \\
\hline Empty follicular openings & 26 & $60 \%$ \\
\hline Irregular white dots & 8 & $19 \%$ \\
\hline Pilli torti & 3 & $7 \%$ \\
\hline Trichorhexis nodosa & 2 & $5 \%$ \\
\hline
\end{tabular}

Table 3: Tricoscopic findings.

\begin{tabular}{|c|c|c|}
\hline$<\mathbf{1 0}$ years of evolution & Number & Percentage \\
\hline $\begin{array}{c}\text { Preserved sebaceous } \\
\text { glands }\end{array}$ & 6 & $75 \%$ \\
\hline Decrease in terminal hairs & 8 & $100 \%$ \\
\hline Fibrotic fibrous tracts & 3 & $37 \%$ \\
\hline $\begin{array}{c}\text { Sparse lymphocytic } \\
\text { inflammation }\end{array}$ & 7 & $87 \%$ \\
\hline
\end{tabular}

Table 4: Findings in patients wiht less than 10 years.

\section{Discussion}

The prevalence of traction alopecia is estimated to range from $1 \%$ to $37 \%$. Affects one-third of African American women, and the Hispanic population being the second most frequent ethnic group for this type of hair loss [3,6]. In Mexico, a study on the frequency of types of alopecia reported that traction alopecia was the second cause of consultation due to hair loss, representing $22 \%$ of the sample studied [7].

The location of the patchy areas in traction alopecia depends on the hairstyle used. When it is secondary to the use of braids, cornroads, dreadlocks, hair clips or pins, it can appear on any area of the scalp, commonly observed, linear or geometric paths [8]. A horseshoe pattern located in the occipital region secondary to the use of artificial curtain hair extensions has been described. In the case of the ponytails, buns and turbans, the most common presentation is a "marginal pattern", located in the bitemporal region of the scalp (frontal, temporoparietal and even occipital hairlines) $[1-3,5]$.

In our patients, the most common localization was the bitemporal region, above the preauricular region, followed by the frontal region and finally in the occipital region. We observed the called "fringe sign" (preservation of numerous terminal hairs on the anterior border) in all the patiens. This finding is reported in the literature in 85 to $100 \%$ of cases and its presence should be considered a highly suggestive clinical finding of AT [4].

Traction alopecia has been considered a biphasic disease, with an early (reversible) and a late stage (scarring alopecia). In the first stage called "traction folliculitis", condition located in the areas of maximum traction and characterized by folicular papules and pustules, with decreased capillary density and occasionally alopecic patches [9]. These lesions are caused by irritation and damage secondary to the traction exerted on the hair, which loosens and injures the internal and external root sheath of the hair follicle, a process that is accompanied by mild inflammatory infiltrates $[10,11]$.

It has been observed that if the traction is very intense, the follicle can be plucked and the dermal papilla can even be extracted, wih permanently damaging and losing of the regenerative capacity the hair. This would explain the irreversible nature of alopecia in some cases [12].

Traction folliculitis is most often seen in girls and teenagers. In our patients it was observed in the youngest patients (16 to 22 years old). These patients were generally combed by a third person, the mother or caregiver.

Regarding trichoscopic characteristics, has been described the presence of broken hair, decreased capillary density and hairiness, peripheral erythema, and peripillary casts $[13,14]$. Peripillary cast are whitish concretions, that surround the hair fiber. They are usually located a few distance from the scalp and are considered a product from the internal and external root sheath [15]. They occur in 20 to $80 \%$ of cases, being more frequent in early and active stages and decreasing in the late stage. Its observation in the initial consultation is suggestive of the diagnosis and its persistence over time is an indication that traction continues and therefore alopecia can progress [15,16]. Polat, et al. studied 25 women with a clinical diagnosis of AT, and reported:

1. Decreased capillary density, diameter variability and hairy hair in $100 \%$ of their patients, these characteristics were identical in our patients. Yellow dots in 68\%, loss of follicular openings in $78 \%$, yellow dots and broken 
hair in $68 \%$, black dots and peripheral erythema in $48 \%$, peripillary cast in $28 \%$

2. In our study, the most frequent tricoscopic finding was the peripillary cylinders in $81 \%$, well above that described by Polat. The second most frequent finding was peripheral brown pigmentation (77\%), it must be considered that all the patients had skin phototypes III or IV. Black spots and broken hair were found in 53 and $51 \%$ respectively.

The histopathological findings of traction alopecia depend on the stage of the disease. In early stages, the findings are similar to those of trichotillomania, presenting a normal number of hair follicles, an increase in the count of catagen / telogen follicles, pigment casts, trichomalacia, alteration of follicular architecture and peripheral / intrafollicular hemorrhage $[4,6]$. Being a result of the mechanical trauma suffered by the hair follicle. The presence of a mild inflammatory infiltrate has also been described in early stages of the disease [4]. An increase in the number of catagen / telogen follicles and the presence of lymphohistiocytic inflammatory infiltrate in the upper segment were observed in our patients. It is believed that if the hair follicle undergoes constant traction for a long period, chronic peripheral inflammation develops, causing scarring alopecia [17].

In late stages, the disease shows characteristics of scarring alopecia. Loss of terminal follicles can be observed, which are replaced by fibrous tracts as well as a decrease in the terminal / hairy ratio, due to the fact that the total number of hair follicles is conserved [18]. The decrease in the terminal / vellous ratio was observed only in $50 \%$ of our patients. At the same time, preservation has been described in the sebaceous glands, although reduced in size, which has been considered as an indicator of progression to scarring alopecia. Van Gieson staining and Masson's trichrome staining have identified loss of elastic fibers and peripheral fibrosis respectively. Another finding is the presence of bare hairy stems, in which only the hair shaft is observed, due to the destruction of the follicular epithelium [19]. This data is observed in the late stage of other scarring alopecia and was observed in one of our cases [17-19].

\section{Conclusion}

In the authors experience, traction alopecia is more frequent than reported in the literature, we consider that in most cases it is confused with other causes of alopecia that are located in the marginal line, such as fibrosing frontal alopecia, effluvium. telogen and alopecia areata ofiacea. Although we cannot rule out that it may be accompanied by other hair disorders.
The diagnosis is clinical and requires careful observation of the clinical characteristics: distribution, shape, presence of the fringe sign and even the hairstyle worn by the patient at the time of the consultation. We propose an easy way to identify this disease, we recomended to use the $3 \mathrm{~T}$ technique of alopecia: (Traction, Time of evolution and hairstyle Type).

It is important to disclose its clinical and trichoscopic characteristics among dermatologists, health personnel and even hairdressers in order to carry out a timely detection. As well as massive information and awareness campaigns in schools and mothers of families in relation to the hairstyle in order to prevent this condition.

\section{References}

1. Khumalo N, Jessop S, Gumedze F, Ehrlich R (2007) Hairdressing and the prevalence of scalp disease in African adults. Br J Dermatol 157(5): 981-988.

2. Mualla P (2017) Evaluation of clinical signs and early and late trichoscopy findings in traction alopecia patients with Fitzpatrick skin type II and III: a single-center, clinical study. International Journal of Dermatology 56(8): 850-855.

3. Khumalo NP, Jessop S, Ehrlich R (2006) Prevalence of cutaneous adverse effects of hairdressing: a systematic review. Arch 86 Dermatol 142(3): 377.

4. Samrao A, Price VH, Zedek D, Mirmirani P (2011) The "Fringe Sign" - A useful clinical finding in traction alopecia of the 92 marginal hair line. Dermatol Online J 17: 1 .

5. Muñoz O, Vaño S (2016) Bitemporal hair loss related to traction alopecia. Dermatology Online Journal 22(9): 13030.

6. Billero V, Miteva M (2018) Traction alopecia: the root of the problema. Clinical and Cosmetic and Investigational Dermatology 11: 149-159.

7. Morales-Miranda AY, Morales TML (2018) Most frequent alopecias in a third-level dermatological center. Dermatol Rev Mex 62(3): 216-220.

8. Haskin A, Aguh K (2016) All hairstyles are not created equal: What the dermatologist needs to know about black hairstyling practices and the risk of traction alopecia. J Am Acad Dermatol 75(3): 606-611.

9. Fox GN, Stausmire JM, Mehregan DR (2007) Traction folliculitis: an underreported entity. Cutis 79(1): 26-30. 


\section{Clinical Dermatology Open Access Journal}

10. Dawber RP (1979) Hair casts. Br J Dermatol 100(4): 417-421.

11. Rollins TG (1961) Traction follicultis with hair casts and alopecia. Am J Dis Child 101(5): 639-640.

12. Sperling LC (1991) Hair anatomy for the clinician. J Am Acad Dermatol 25(1): 1-17.

13. Miteva M, Tosti A (2012) Hair and scalp dermatoscopy. J Am Acad Dermatol 67(5): 1040-1048.

14. Mubki T, Rudnicka L, Olszewska M, Shapiro J (2014) Evaluation and diagnosis of the hair loss patient: part II. Trichoscopic and laboratory evaluations. J Am Acad Dermatol 71(3): 431.e1-431.e11.

15. Tosti A, Miteva M, Torres F, Vincenzi C, Romanelli P (2010) Hair casts are a dermoscopic clue for the diagnosis of traction alopecia. Br J Dermatol 163(6): 1353-1355.
16. Mathur M, Acharya P, Karki A, Shah J, Nisha KC (2019) Tubular Hair Casts in Trichoscopy of Hair and Scalp Disorders. Int J Trichology 11(1): 14-19.

17. Tanus A, Oliveira CC, Villarreal DJ, Sanchez FA, Dias MF (2015) Black women's hair: the main scalp dermatoses and aesthetic practices in women of African ethnicity. An Bras Dermatol 90(4): 450-65.

18. Bernárdez C, Molina-Ruiz AM, Requena L (2015) Histologic features of alopecias-part I: nonscarring alopecias. Actas Dermosifiliogr 106(3): 158-167.

19. Ngwanya RM, Adeola HA, Beach RA, Gantsho N, Walker CL, et al. (2019) Reliability of Histopathology for the Early Recognition of Fibrosis in Traction Alopecia: Correlation with Clinical Severity. Dermatopathology (Basel) 6(2): 170-181. 